\title{
Readmission rate according to medication state of previous admission. (Is oral medication effective enough for preventing multiple readsmission?)
}

\author{
Hye Ok Kim', Gi Hyeon Seo', Boung Chul Lee ${ }^{2}$ \\ I Health Insurance Review and Assessment Service, South Korea \\ 2 Department of Psychiatry, Hangang Sacred Heart Hospital, Hallym University Medical Center, \\ Seoul, South Korea
}

We investigated readmission rate of schizophrenia population with various admission and its readmission rate ratio comparing with no medication, medication and long acting injections.

A importance of medication to prevent admission in maintenance phase of Schizophrenia cannot be emphasized enough. However, some patients experience symptom aggravation even during medication period and sometime need readmission to control. Long acting injections were developed to prevent readmission especially to poor compliance patients.

But in randomized controlled trial the evidence is not strong enough to its clear benefit of preventing acute aggravation is Schizophrenia. We think this limitation is due to strict research setting which cannot represent practical treatment environment. Because, usually RCT participating patients are highly compliant.

We analyzed 2008-2016 claim data in South Korea to see readmission rate according to medication state to verify different effect of oral medication and long acting injection. We collected Schizophrenia (F20, F25) as main diagnosis with admission duration from 7 days to 120 days.

A Total 75,274 patient data were analyzed. Patients with at least twice multiple admission were divided as previous medication state (no medication, medication and long acting injection), readmission rate were compared.

Readmission rate comparing with previous medication to oral medication was 0.75 . And previous oral medication to long acting injection was 0.52 . Which means with previous admission during oral medication patients, oral medication after that discharge could prevent only $25 \%$ of readmission. However, its rate increases to $48 \%$ while taking long acting injections.

We think long acting injection has clear benefit over multiple admission patients from preventing readmission.
Fig 1. Readmission of Schizophrenia patients after discharge with multiple admission history

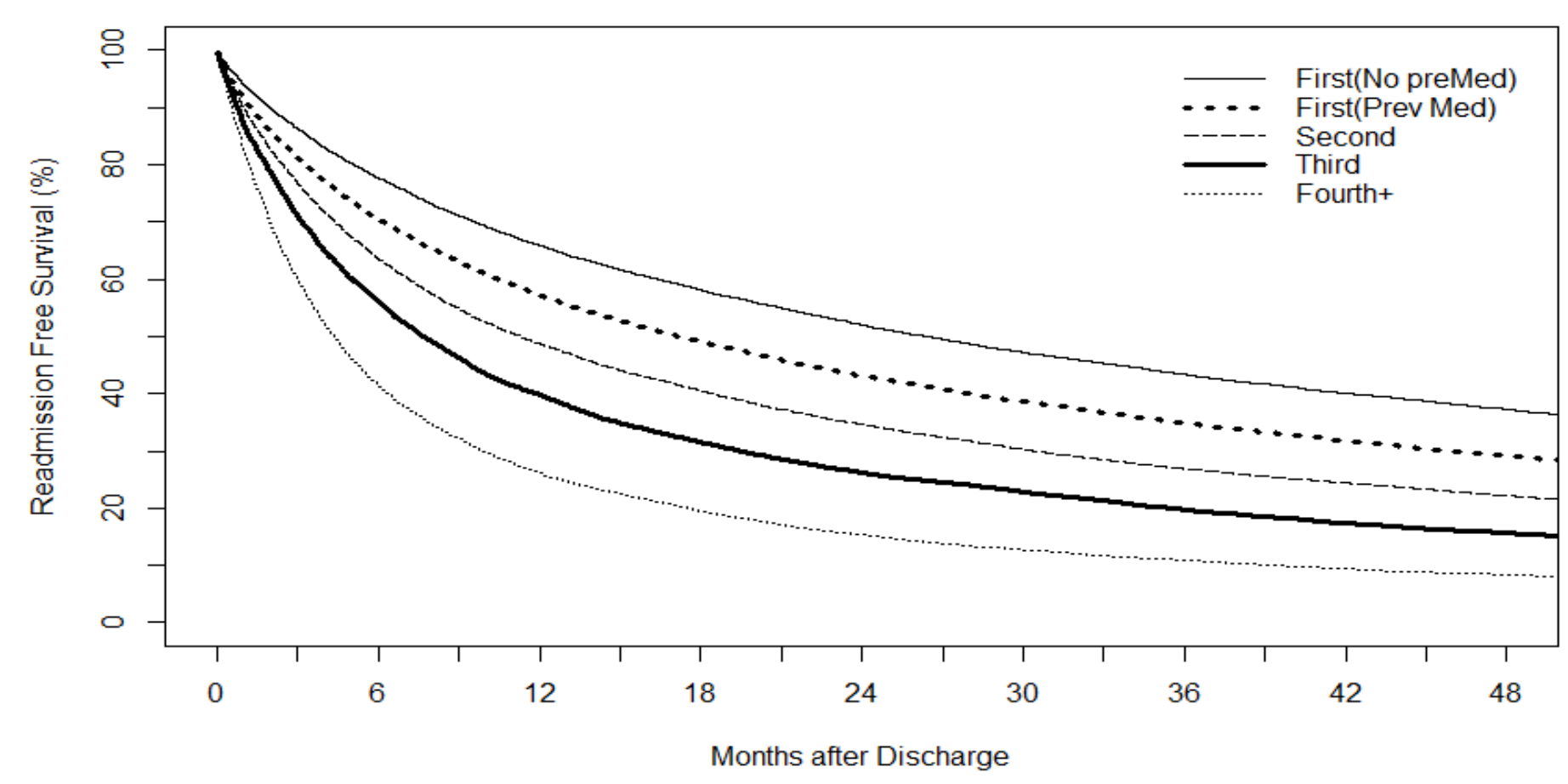

Fig 2. Readmission of Schizophrenia based on medication state at third admission.

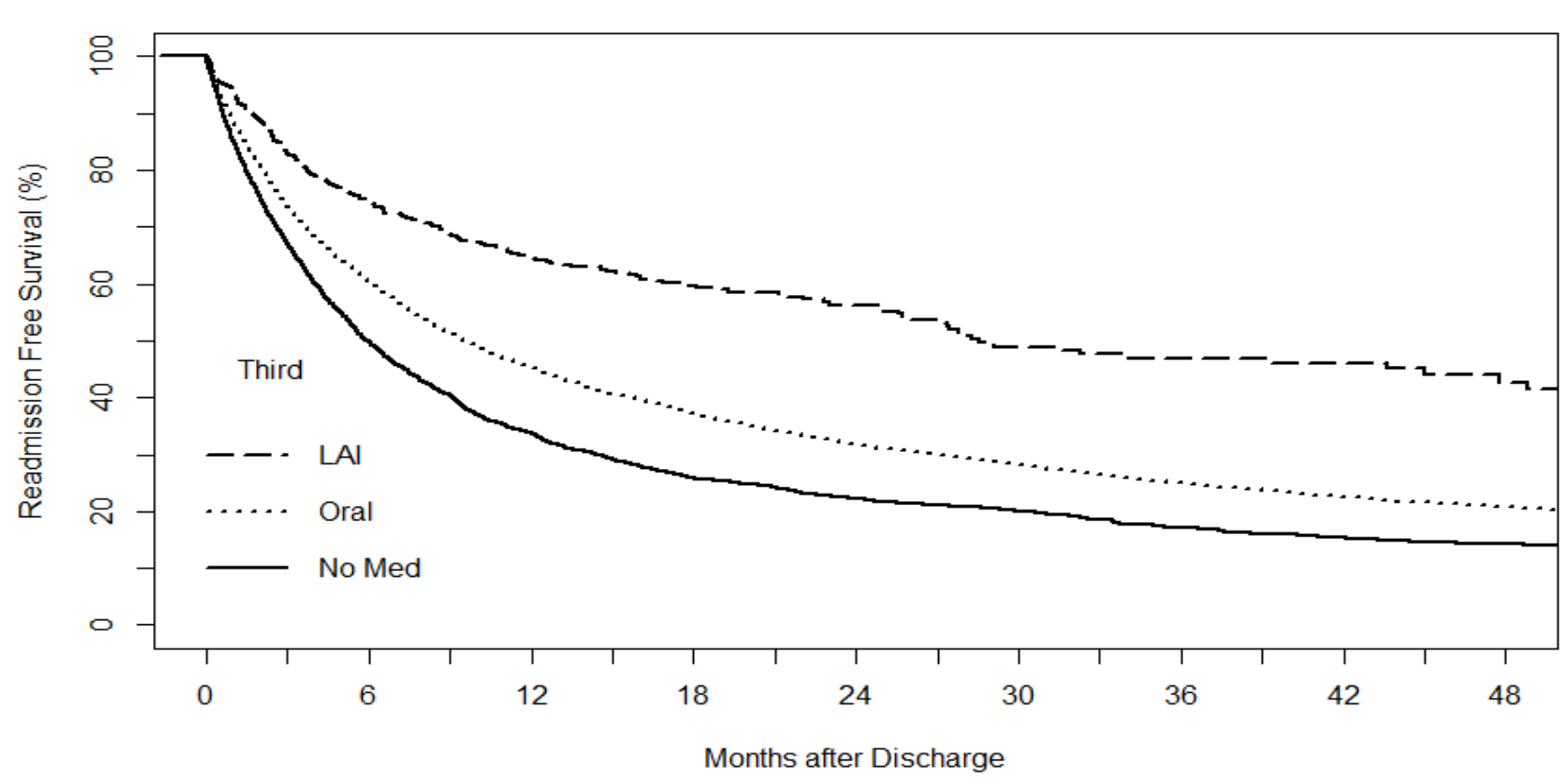

* LAI : long acting injection (including injection with oral medication), Oral : any oral antipsychotic medication, No Med : no medication

Ref )

Tiihonen J, Mittendorfer-Rutz E, Majak M, et al. Real-World Effectiveness of Antipsychotic Treatments in a Nationwide Cohort of 29823 Patients With Schizophrenia. JAMA psychiatry 2017;74(7): 686-93

Kishimoto T, Robenzadeh A, Leucht C, et al. Long-acting injectable vs oral antipsychotics for relapse prevention in schizophrenia: a metaanalysis of randomized trials. Schizophrenia bulletin 2014;40(1): 192-213 